\title{
Effects of Stricter Management Guidelines on Return-to-Competition Timeframes Following Concussion in Professional Australian Rules Football: An Exploratory Analysis
}

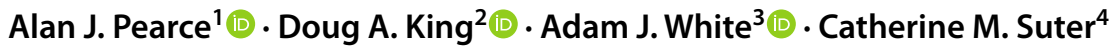 \\ Accepted: 3 May 2021 / Published online: 15 May 2021 \\ (C) The Author(s), under exclusive licence to Springer Nature Switzerland AG 2021
}

\begin{abstract}
Background Management of concussion remains a serious issue for professional sports, particularly with the growing knowledge on the consequences of repetitive concussion. One primary concern is the subjective assessment of recovery that dictates the time until a concussed athlete is returned-to-competition. In response to this concern, the Australian Football League (AFL) changed its policy in 2020 such that medical clearance for return-to-competition was extended from 1 day, to a minimum of 5 days, prior to the next scheduled match.

Objective We sought to explore the impact of the AFL policy change by asking whether time to return-to-competition after concussion was increased in the 2020 season relative to previous years.

Methods Retrospective data on injury and return-to-competition were sourced from publicly available tables published by the AFL. Our primary exploration compared the number of matches missed and the number of days missed in concussed players across 2017-2020 inclusive, with secondary exploration analysing the proportion of players returning to play 12 days or longer.

Results Analysis of data from 166 concussed players revealed no increase in the number of matches missed in 2020 relative to previous years as would have been expected from an extended recovery protocol. Comparing 2020 relative to 2017-2019, we found that there was an overall moderate reduction in median time to return-to-competition (RTC) in 2020 (10 vs 13 days, respectively $d=-0.345)$ and a significant reduction in players taking more than 12 days to $\operatorname{RTC}(p=0.046)$.

Conclusion This exploratory study demonstrates that clubs may not have followed policy change around concussion management designed to increase time to RTC. Ongoing auditing is required to ensure player clearance meets policy goals, highlighting the need for objective measures for RTC after concussion.
\end{abstract}

Alan J. Pearce

alan.pearce@latrobe.edu.au

1 College of Science, Health and Engineering, La Trobe University, Kingsbury Drive, Bundoora, Melbourne, VIC, Australia

2 Faculty of Health and Environmental Science, Sports Performance Research Institute New Zealand (SPRINZ), Auckland University of Technology, Auckland, New Zealand

3 Department of Sport, Health Sciences and Social Work, Faculty of Health and Life Sciences, Oxford Brookes University, Oxford, UK

4 Department of Neuropathology, Royal Prince Alfred Hospital, Sydney, Australia 


\section{Key Points}

In 2020, the Australian Football League (AFL) changed their concussion management policy to increase the time between when a player could be medically cleared prior to their next competitive match to five days. This should extend the number of days between concussion and return-to-competition to a minimum of 12 days.

Using publicly available data, this study reports on the average number of matches missed and the number of day's missed between a player's reported concussion and their first competitive match.

Comparison of matches and days missed between 2017 and 2019 (old policy) and 2020 (new policy) demonstrated that clubs were not following the policy as carefully as they should. The data show that players were rested for shorter periods in 2020.

\section{Introduction}

The international consensus statement on the management of concussion in sport (the Berlin statement) [1] promotes a strategy whereby an athlete recovering from concussion is exposed to graduated stages of increasing activity, known as the 'return-to-sport' strategy (Fig. 1a). This is a six-stage protocol in which players can only pass from one stage to the next as long as concussion symptoms are resolving. Following removal from play after a suspected concussion event, the athlete is medically assessed by the team doctor. If diagnosed with a concussion, the athlete is no longer able to participate in the match. Following this, a minimum rest time of 24-48 $\mathrm{h}$ post-concussion is recommended prior to beginning the return-to-sport steps, and the minimum recommended interval between stages is no less than $24 \mathrm{~h}$. Clearance for return-to-sport is a medical judgement made by the team doctor, in consultation with the coach and player. Clearance can only occur if the athlete declares that they are symptomfree, and the treating physician deems the athlete is safe to return-to-sport. This includes return to full contact training prior to competition play [1].

This seems a sensible policy; however, there are criticisms of this approach, the most prominent of which is the confidence placed in players' self-reporting of symptoms for

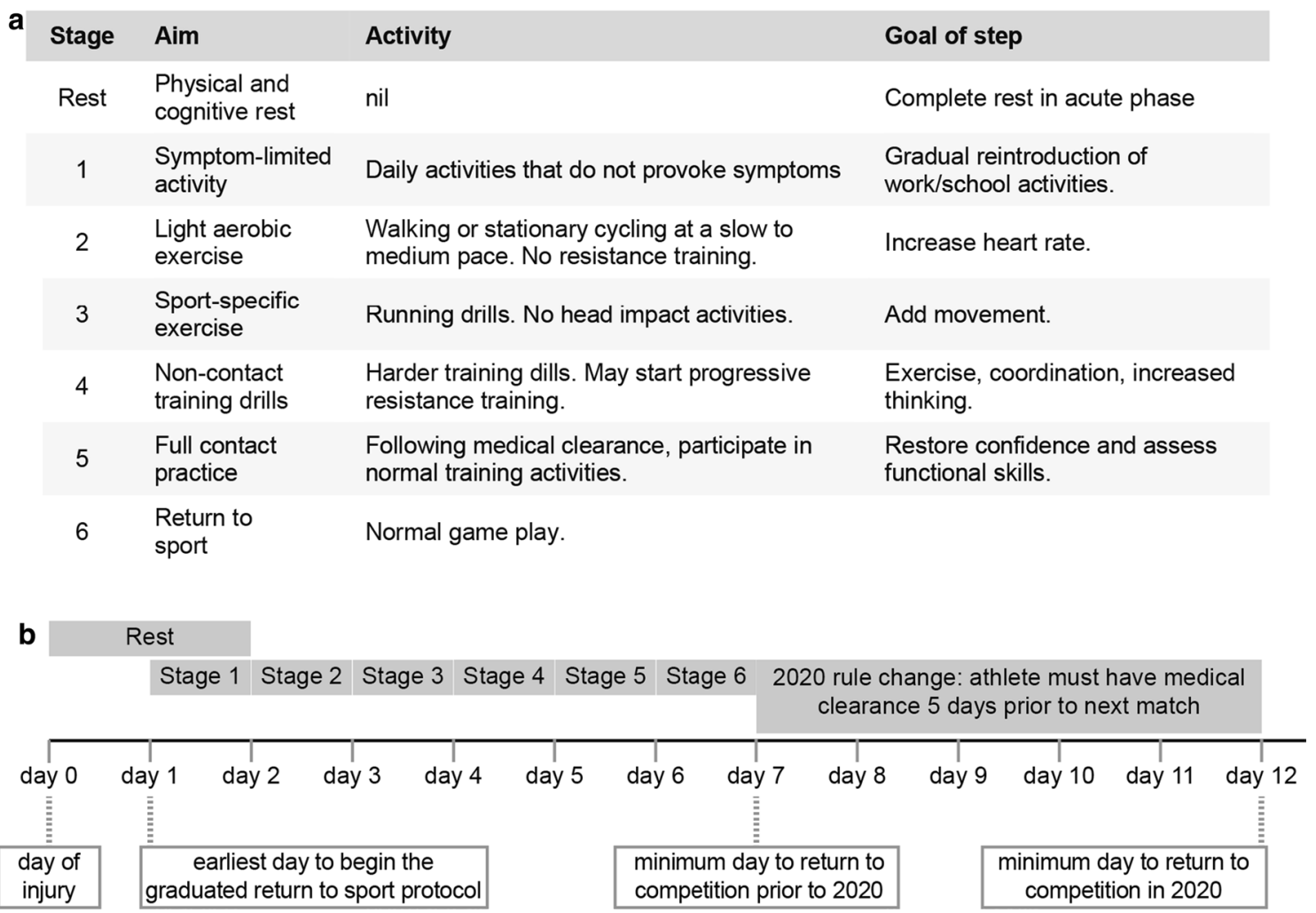

Fig. 1 Summary of the return-to-sport strategy (a) as detailed in [1] and schematic of the expected return-to-competition timeframes (b) 
progression through the various stages. This subjectivity in assessing recovery is further complicated by the fact that after a concussion, symptom resolution and an individual's physiological functioning are not necessarily correlated [2]. Consequently, there are ongoing concerns that athletes are returning to competition prematurely after a concussion, increasing both the risk of further injury, and the likelihood of persistent post-concussion symptoms [1, 3-6].

In an attempt to address these concerns, the Australian Football League (AFL), who adhere to the protocols based on the Berlin statement [7], recently changed their concussion management guidelines. Prior to the 2020 season, medical clearance for return-to-competition play (returnto-competition) stipulated AFL players be cleared by the team doctor prior to the next match; in theory, a player could be cleared the day before (or even on the day of) the next match. Now in 2020, the AFL have introduced a stricter policy, mandating medical clearance occur at least five days prior to the team's next scheduled competition match $[8$, 9]. The AFL proposed this policy change would make it more difficult for players to return-to-competition the week after sustaining a concussion. While these changes fall short of mandating a compulsory break in play for at least one competition match (i.e., 12 or more days), if followed in lock-step with the consensus protocol, the new rules should effectively result in at least 12 days between concussion and the next match (Fig. 1b).

The effectiveness of this strategy to increase the time between concussion and return-to-competition can be assessed objectively, because the AFL publishes detailed information on injuries and play via its website. We interrogated this publicly available data to explore whether the new concussion management policy increased the time players were out of the game in the number of matches missed, and the overall number of days missed between concussion and return-to-competition. We also explored the data to investigate the proportion of players returning to play having not missed a match or having missed 12 or more days of play after a concussion.

\section{Methods}

Concussion injury and fixture data were collected from www.afl.com.au/matches/injury-list and www.afl.com.au/ fixture, respectively. As all player data were in the public domain, human ethics approval for this study was considered unnecessary (Health and Disability Ethics Committee NZ). Inclusion/exclusion criteria and our analytical strategy are shown in Fig. 2. Players were included if they were: (1) AFL listed; (2) played at least one match in the AFL; (3) were included in the Injury List with concussion and; (4) either returned-to-competition within the season or were indefinitely removed from play because of concussion.

Players who returned-to-competition after concussion had the number of matches missed recorded, and also the days between the concussion injury and the next match played. We surveyed this data for the years of 2017-2020 inclusive. Due to the COVID-19 pandemic, the number of rounds in the 2020 competition was reduced from 23 rounds in 2017-2019 seasons, to 18 rounds in 2020. Consequently, data from 2017 to 2019 on athletes who returned-to-competition following a concussion after round 18 were not included in the analysis.

Following screening of data, statistical comparisons of quantitative data (i.e., the number of matches/days missed) across all years were made using the nonparametric independent-samples Kruskal-Wallis test; the Mann-Whitney $U$ Test was used to compare 2020 to all other years combined. Contingency tables were used to compare categorical data (i.e., proportion of players with any/no matches missed, and proportion with more/less than 12 days missed) with chi-square tests for comparison between 2020 and all other years, and Fisher's Exact test for 2020 versus all years combined. Data are presented as mean and $95 \%$ confidence intervals (CI) for descriptive data, and median and interquartile ranges for matches and days missed after concussion. Where appropriate, effect size (Cohen's $d$ ) was used to calculate effect differences between groups $(\leq 0.2=$ small; $0.31-0.8=$ medium; $\geq 0.81=$ large) [10]. Kaplan-Meier estimators were used to determine return-to-competition probabilities and these were compared using the Mantel-Cox log rank test. For all tests, an alpha value of 0.05 or less was considered significant.

\section{Results}

As outlined above, the timeline for the 2020 season was disrupted by COVID-19 such that meaningful comparisons can only be made considering 18 rounds of play. We began comparisons by compiling the details of concussions sustained across the 18 rounds of 2020 , along with first 18 rounds of each of the 2017 to 2019 seasons. The frequency of concussion was similar across the years (mean $41.5 \pm 5.2$, range 36-48). Table 1 shows the descriptive return-to-competition statistics for each year.

In our primary exploration involving quantitative analysis of 143 players who sustained a concussion and subsequently returned to play, we found that the median number of matches missed in 2020 was one; the median matches missed in 2018 and 2019 was also one, and in 2017 the median was zero (Fig. 3a). There was no difference in the distribution of the number of matches missed between all years ( $p=0.246$; Fig. 3a), and also no difference within 
Fig. 2 Inclusion/exclusion criteria and analysis strategy
Identification

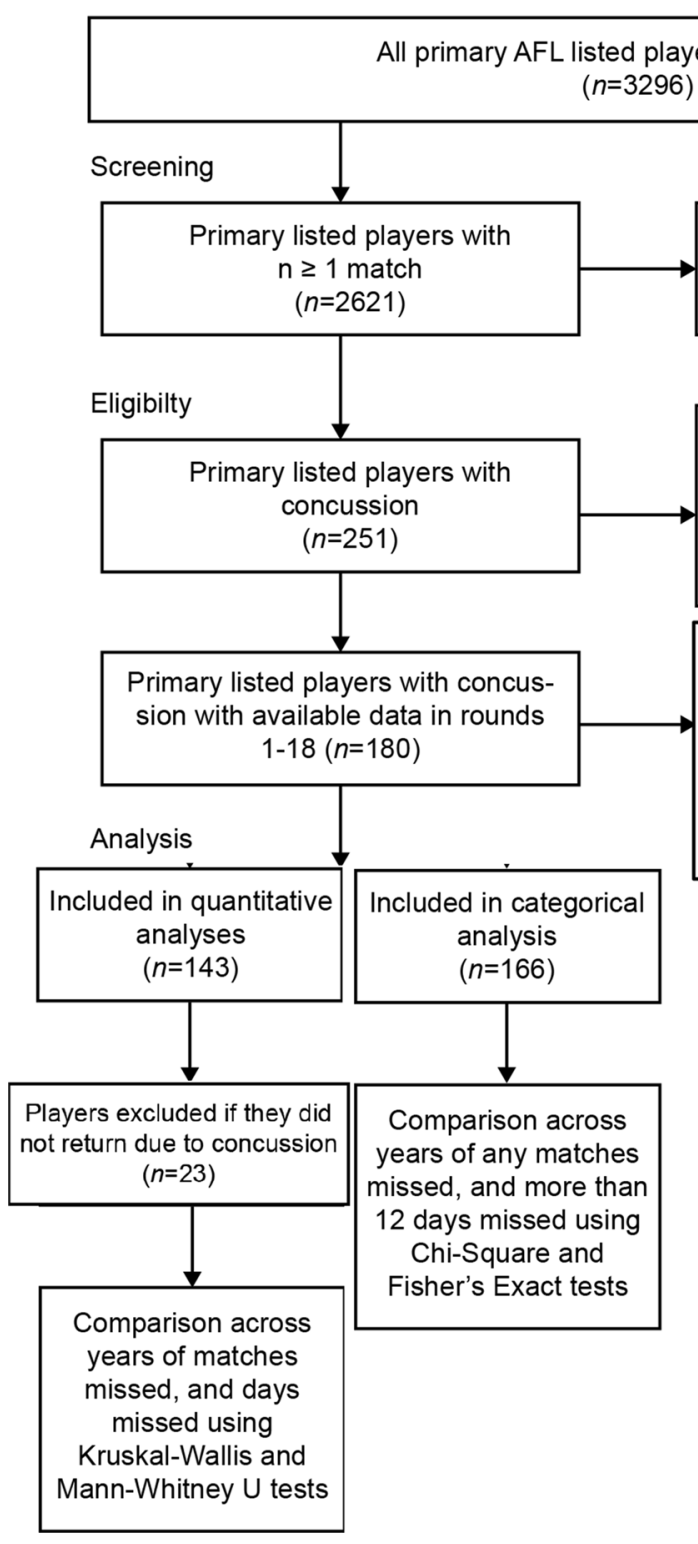

Players excluded if not playing at least one AFL match $(n=675)$
Players excluded if not returning to season because of reason other than concussion or no record of $A F L$ following recovery $(n=71)$

Players excluded if injury sustained in round 18 or later $(n=37$; quantitative analysis)

Players placed on indefinite injury list after concussion $(n=23$; categorical analysis)
Table 1 Descriptive statistics for concussion and return-tocompetition in professional AFL from 2017 to 2020

\begin{tabular}{|c|c|c|c|c|}
\hline & 2017 & 2018 & 2019 & 2020 \\
\hline Players concussed in rounds $1-18 n$ & 43 & 48 & 39 & 36 \\
\hline Sum of matches missed ${ }^{\mathrm{a}} n$ & 29 & 61 & 55 & 27 \\
\hline Mean matches missed $n( \pm 95 \mathrm{CI})$ & $0.8(0.4)$ & $1.5(0.6)$ & $1.7(0.5)$ & $0.8(0.4)$ \\
\hline Total time to RTC ${ }^{\mathrm{a}}$, days & 453 & 710 & 618 & 370 \\
\hline Mean time to $\mathrm{RTC}^{\mathrm{a}}$, days $( \pm 95 \mathrm{CI})$ & $12.9(2.9)$ & $16.9(4.3)$ & $18.7(3.6)$ & $11.2(2.0)$ \\
\hline Players not returning to season ${ }^{\mathrm{b}} n(\%)$ & $8(18.6)$ & $6(12.5)$ & $6(15.0)$ & $3(8.3)$ \\
\hline Players not missing a match after concussion $n(\%)$ & $18(41.9)$ & $18(37.5)$ & $11(28.2)$ & $16(44.4)$ \\
\hline Players missing 12 or fewer days after concussion $n(\%)$ & $18(41.9)$ & $22(45.8)$ & $13(33.3)$ & $21(58.3)^{*}$ \\
\hline
\end{tabular}

$R T C$ return-to-competition; SD, standard deviation; *significantly different to all other years combined $(p=0.046)$

${ }^{a}$ Only counted for those players returning to play in that season

${ }^{\mathrm{b}}$ Not returning because of concussion 
Fig. 3 Matches missed after concussion $(\mathbf{a}, \mathbf{b})$ and time to return-to-competition $(\mathbf{c}, \mathbf{d})$ across the years indicated. Only players who did return to the same season are included. Boxand-whiskers plots show the median (red lines) and the interquartile range (IQR; boxes); whiskers show $1.5 \times$ the IQR; outliers are plotted as circles (e, f) Average matches missed (e) and average time to return-tocompetition (f) plotted as group mean \pm 95 CI. RTC: return-tocompetition; $2017 n=35,2018$ $n=42,2019 n=33,2020 n=33$
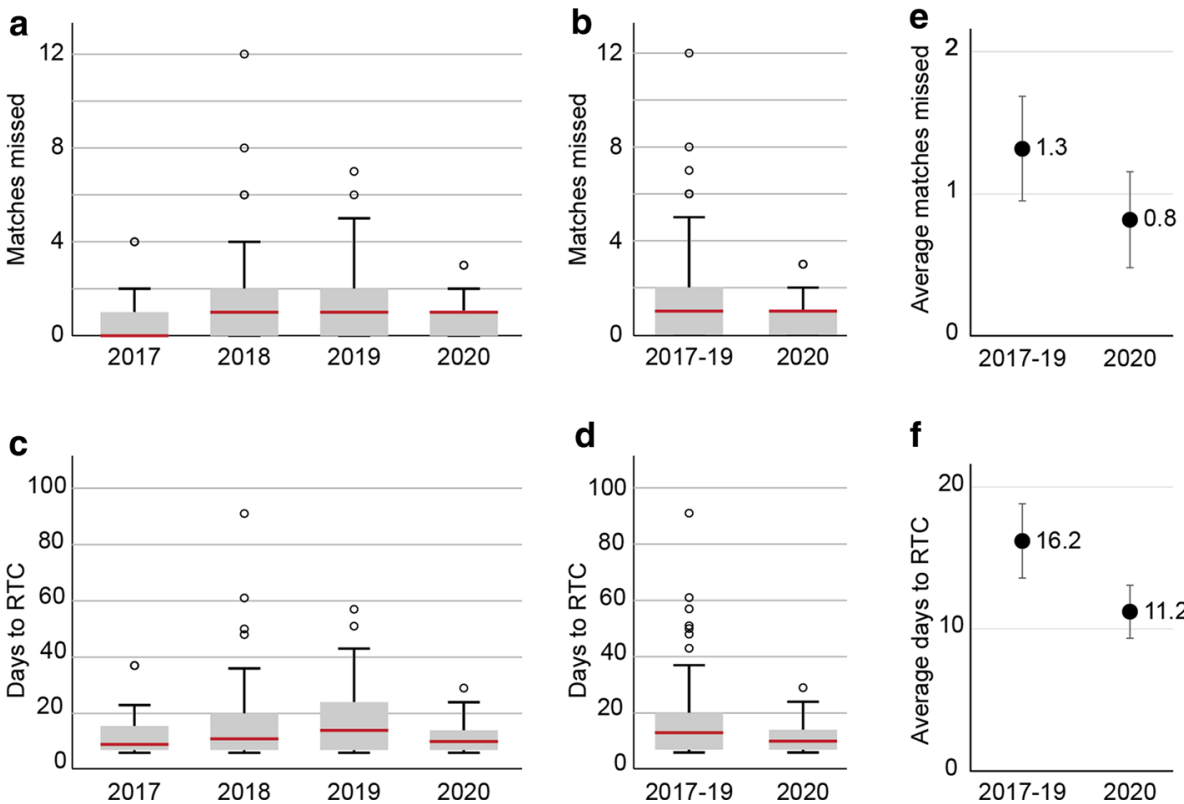

f

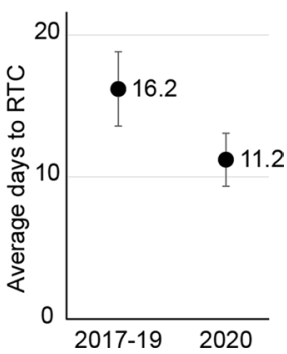

the years of the previous return-to-competition policy $(p=0.176)$. We thus considered 2017-2019 (previous policy) as a group and found that while the median remained at one in each group (Fig. 3b), there was a reduction in the mean number of matches missed in 2020 relative to the previous years combined ( 0.8 vs 1.3 ; Fig. $3 \mathrm{c}$ ). Although this reduction was not statistically significant, the effect size was moderate $(d=-0.345)$. This finding was unexpected as the new policy was designed to increase the time to return-tocompetition after a concussion.

We considered that these results might be attributable to changes in match scheduling because of COVID-19; some matches in 2020 were delayed, or brought forward. We thus compared the time to return-to-competition (in days) after concussion. We found that across all rounds that there were no significant differences observed in mean return-to-competition times, although 2020 had the least number of days missed across all years (Table 1; Fig. 3d). There were no significant differences observed between years within the old policy, so we again considered 2017-2019 as a group, and compared the time to return-to-competition to 2020 . These results show that there was a reduction in the both the median and mean time to return-to-competition in 2020 compared to the previous years (median 13 vs 10 days; mean 11.2 vs 16.2 days (Fig. 3e, f). While not reaching significance $(p=0.200)$, the effect size was again moderate $(d=0.524)$.

There were 23 additional players across the 4 years who sustained a concussion and were subsequently sidelined indefinitely because of their concussion (Table 1). These players could not be included in the primary quantitative analysis above but can be included in a secondary categorical analysis of return-to-competition. We categorised all 166 players as either having missed no match after their injury, or one or more matches. We found that the proportion of players who did not miss a match after concussion was $42 \%$ in 2020; however, there was no significant difference between years, or between 2020 and all years combined (Fig. 4a).

The new 2020 policy should result more players returning to competition play 12 days or later after their concussion injury [9] (Fig. 1b). As part of this secondary analysis, we therefore also characterised players on this basis. In 2020, only $42 \%$ of players had more than 12 days between their concussion and return-to-competition (Fig. 4b). This means that more than half of all players (58\%) who sustained a concussion in 2020 were back on the field within 12 or fewer days. Contrary to expectations, the proportion of players taking more than 12 days to return-to-competition was significantly reduced in 2020 compared with previous years $\left(\chi^{2}(1\right.$, $n=166)=3.520$, Fisher's Exact $p=0.046$; Fig. 4b).

The analyses above rely on comparing the distribution of values using nonparametric tests. A more holistic view of the data can be taken by comparing probabilities of returning to competition. We thus generated Kaplan-Meier (KM) plots to show the relative rates of return-to-competition after concussion across the years. Figure 5a shows KM plots for each year individually, with 2020 showing the fastest trajectory for all players returned. When grouping the years of previous policy together, it is clear that 2020 had a significantly faster trajectory to return-to-competition than the years of the previous policy combined (Mantel-Cox log rank test, $p=0.040$; Fig. 4b).

Taken together, these analyses of AFL public data demonstrate that the change in concussion management policy in 2020 has had no effect on the time to return-to-competition. 

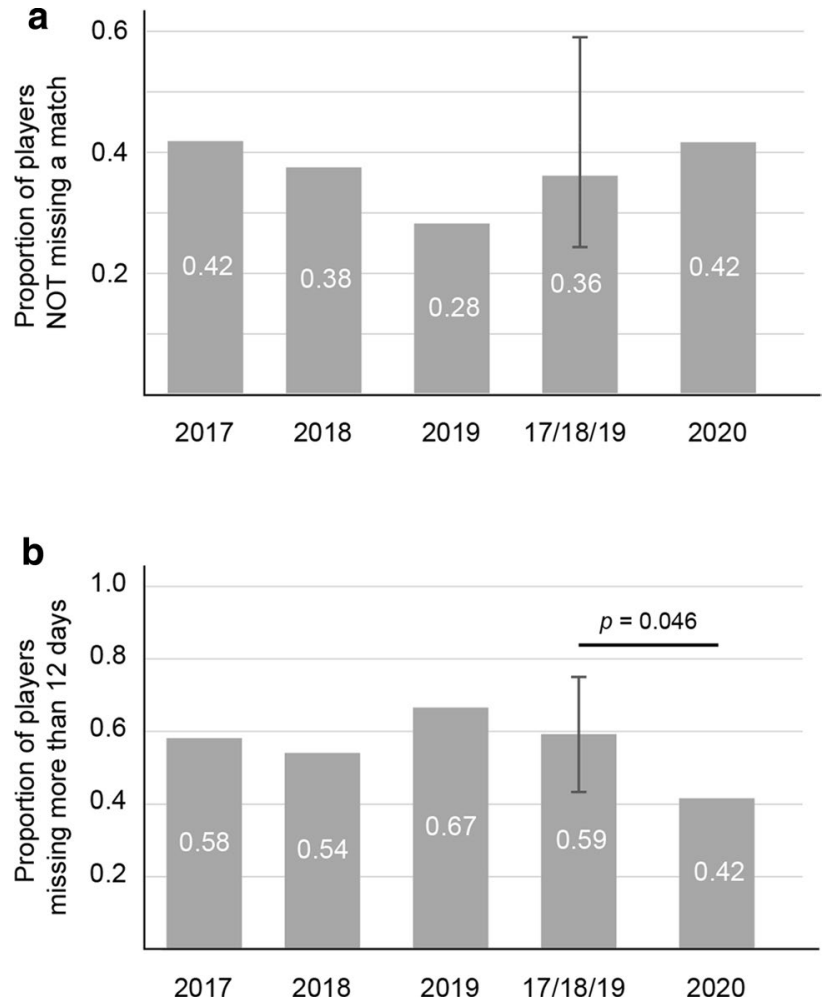

Fig. 4 Bar graphs showing the proportion of all players categorised as (a) having not missed a match after concussion and (b) having missed 12 or more days of play after a concussion. Error bars in grouped data from 2017-2019 represent \pm 95 CI. $2017 n=43,2018$ $n=48,2019 n=39,2020 n=36$

Return-to-competition times in 2020 were found to be shorter than in the previous 3 years.

\section{Discussion}

In this exploratory study, we examined public data to evaluate the effectiveness of an AFL policy change around concussion management. Under the new policy, the minimum interval from concussion to return-to-competition would be expected to increase from 7 to 12 days (to account for the 5 day extra medical clearance timespan) but the data show that this has not occurred. In 2020, the mean number of rounds and days missed after concussion decreased, as opposed to increasing, and the overall trajectory of return-to-competition was faster in 2020 than in the 3 years prior combined. Further we found that a high proportion of concussed players returned-to-competition in 2020 without missing a single game (42\%), or within 12 days (58\%) after concussion.

These 2020 data stand in contrast to the ongoing improvements in the conservative management of concussion in the AFL from 2017 to 2019, as is clear from our data analyses as well as from the official injury report [11]. While the
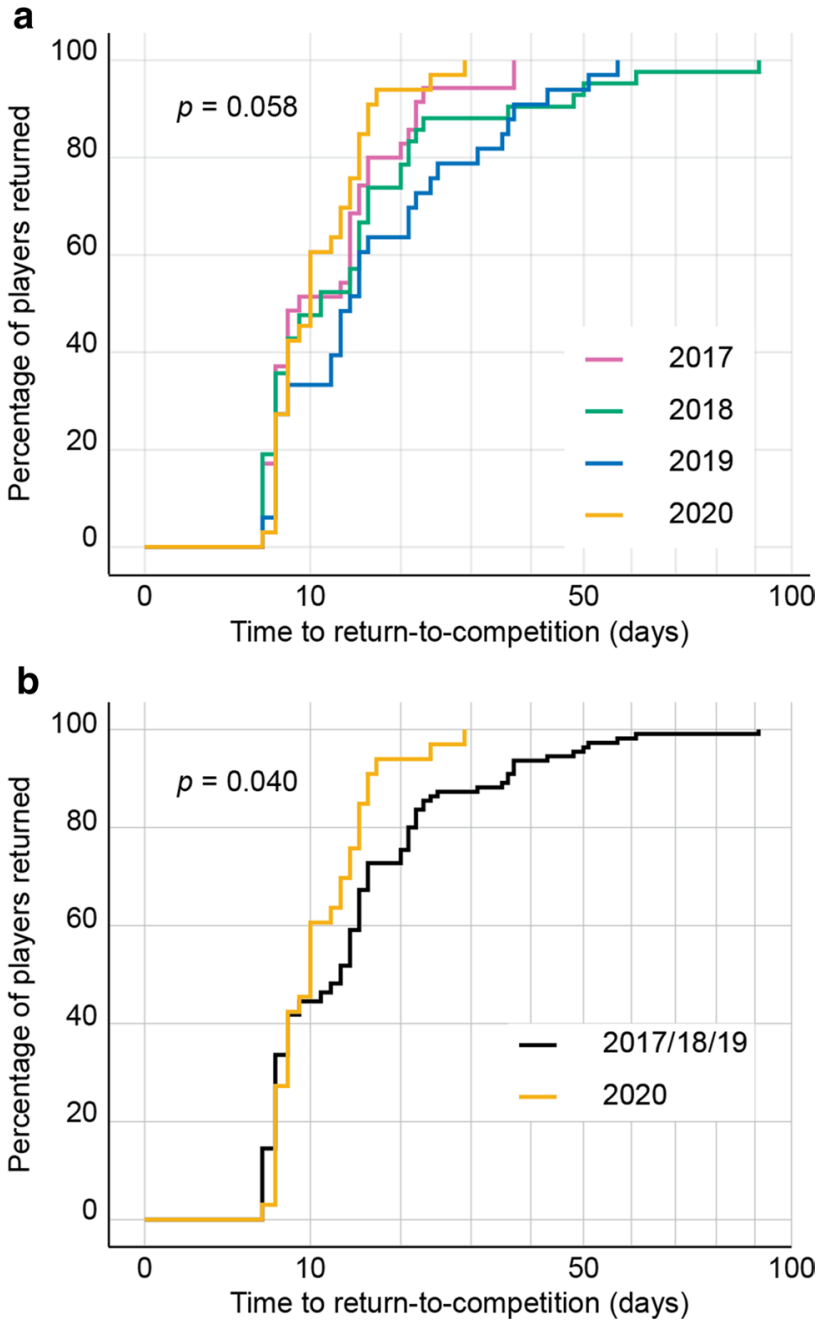

Fig. 5 Time until return-to-competition curves for (a) each year and (b) 2020 vs 2017-2019 combined. Mantel-Cox log rank test reveals 2020 has a significantly faster trajectory to return-to-competition than other years combined $(p=0.040) .2017 n=35,2018 n=42,2019$ $n=33,2020 n=33$

rationale for making the policy change is understood, its lack of effectiveness is more difficult to understand or explain. It may be due, at least in part, to the effects of COVID-19 restrictions and the associated stress in 2020, or it may be due to other factors affecting all years such as concomitant injuries, or the severity of concussion. However in the latter case, concussion is no longer graded by severity [12], so if a player is placed on the injury list with concussion, the minimum respite from play applies irrespective of the number of, and severity of, symptoms reported. In some recent studies, the mean symptom-free duration from concussion injury was 28 days $[13,14]$ yet many players returned to sport in less than half that time.

It is well described that medical staff can be pressured to clear players to return-to-competition as quickly as possible, and this pressure comes from multiple sources including the 
athlete themselves $[15,16]$. In the extraordinary year of 2020 , these pressures may have been amplified, thus leading to a shorter than average return-to-competition time. But this does not excuse the possibility that many players with a concussion in 2020 may have been prematurely returned to the game, risking further injury and the potential post-concussion effects. The reliance on athlete self-reporting on symptom resolution is clearly a 'weak link' in the staged return-to-competition protocol, particularly if an athlete's decision-making capacity is compromised by their concussion injury [17]. Even if this is not the case clinically with an injured athlete, returnto-competition timeframes can be influenced merely by the athlete's knowledge of a change in return protocol, and the desire to return to play. In other words, in some cases, it is possible that knowing when the next match is scheduled, an athlete may be more likely to endorse lower symptoms early on, predicting that they will be cleared by the time of the next scheduled match. Whatever the case, these possibilities caution against overstating the findings as purely an issue for medical practitioners. They also highlight the dire need for standardised objective measures of recovery after concussion. In the absence of such measures, and emerging evidence indicating a longer time course of recovery following a concussion from 14 days to 2 months [18-20], the AFL and other sporting codes should err on the side of caution and mandating that at least one match be missed after suffering a concussion. During review of this paper, the AFL effectively made this change by announcing another policy change for 2021 that mandates a minimum of 12 days rest after a concussion [21].

The reliance of this study on publicly available data provided by the AFL clubs themselves is also a potential limitation of this study. While we are not able to independently verify each case, the data are likely to be accurate as AFL clubs are thought to operate transparently in the listing of relevant injuries in the spirit of the sport, and they are unique among football codes in Australia in this regard. Also, state league data is not readily available to the public to assess whether players were returning to play via this pathway, which could have reduced the number of matches and days missed in previous years (due to COVID-19 this pathway did not operate in 2020). Despite this, we would still have expected to see increased time out of sport in 2020 due to the reduced opportunities for players to return to the AFL via other leagues. Conversely, if players in 2017-2019 were returning within the week to compete in state leagues in spite of the consensus guidelines, this is still of concern with regards to return-tocompetition policies.

\section{Conclusion}

This exploratory study demonstrates that the 2020 AFL policy was not successful in increasing a player's rest and recovery time after concussion. While there is good intention in addressing the ongoing concerns regarding concussion injuries in contact sports, improved monitoring methods during recovery and rehabilitation are necessary to facilitate compliance to any policy, including the new $2021 \mathrm{AFL}$ policy [22]. Adherence to policy based on the best available evidence will ultimately improve players' long-term player health outcomes.

Acknowledgements CMS is supported by the Australian Sports Brain Bank. AJP is funded in part by Sports Health Check charity (Australia) and Erasmus+ strategic partnerships program (2019-1-IE01KA202-051555. No author has any conflict of interest in this work.

Author Contributions AJP conceptualised the study and collected data. CMS and AJP analysed data. All authors interpreted data. AJP and CMS wrote the paper with input from all authors.

Availability of Data and Material All data are available from public websites as outlined in this manuscript.

Code Availability Not applicable.

\section{Declarations}

Funding No specific funding was provided for this research.

Conflict of Interest AJP currently receives partial research salary funding from Sports Health Check charity (Australia) and Erasmus + strategic partnerships program (2019-1-IE01-KA202-051555). AJP has previously received partial research funding from the Australian Football League, Impact Technologies Inc., and Samsung Corporation, and has provided expert reports in concussion legal proceedings. No other author has any declaration of interest.

Ethics Approval Waiver from Health and Disability Ethics Committee NZ.

Consent to Participate Not applicable.

Consent for Publication Not applicable.

\section{References}

1. McCrory P, Meeuwisse W, Dvorak J, Aubry M, Bailes J, Broglio $\mathrm{S}$, et al. Consensus statement on concussion in sport- the 5th international conference on concussion in sport held in Berlin, October 2016. Br J Sport Med. 2017;51:838-47.

2. Pearce AJ, Hoy K, Rogers MA, Corp DT, Davies CB, Maller $\mathrm{JJ}$, et al. Acute motor, neurocognitive and neurophysiological change following concussion injury in Australian amateur football. A prospective multimodal investigation. J Sci Med Sport. 2015;18:500-6. 
3. McPherson AL, Nagai T, Webster KE, Hewett TE. Musculoskeletal injury risk after sport-related concussion: a systematic review and meta-analysis. Am J Sports Med. 2019;47(7):1754-62.

4. Nordström A, Nordström P, Ekstrand J. Sports-related concussion increases the risk of subsequent injury by about $50 \%$ in elite male football players. Br J Sports Med. 2014;48(19):1447-50.

5. Brooks MA, Peterson K, Biese K, Sanfilippo J, Heiderscheit BC, Bell DR. Concussion increases odds of sustaining a lower extremity musculoskeletal injury after return to play among collegiate athletes. Am J Sports Med. 2016;44(3):742-7.

6. Kelly K, Erdal K. Diagnostic terminology, athlete status, and history of concussion affect return to play expectations and anticipated symptoms following mild traumatic brain injury. J Clin Exp Neuropsychol. 2017;39(6):587-95.

7. Davis GA, Makdissi M, Bloomfield P, Clifton P, Cowie C, Echemendia R, et al. Concussion guidelines in national and international professional and elite sports. Neurosurgery. 2020;87:418-25.

8. Chadwick T, Valencich G. AFL set to crack down on concussion with new recovery policy. Seven News. 2020. https://7news.com. $\mathrm{au} / \mathrm{sport} / \mathrm{afl} /$ afl-set-to-crack-down-on-concussion-with-new-recov ery-policy-c-705834. Accessed 19 Feb 2020.

9. McClure S. War brewing over AFL's concussion protocol. The Age. 2020. https://www.theage.com.au/sport/afl/warbrewing-overafl-s-concussion-protocol-20200219-p542fa.html. Accessed 19 Feb 2020.

10. Cohen J. Statistical power analysis for the behavioral sciences. Hillsdale: Erlbaum; 1988

11. Australian Football League. Statement: 2019 AFL injury report.2020. https://www.afl.com.au/news/515940/statement2019-afl-injury-report. Accessed 23 Oct 2020.

12. Giza CC, Kutcher JS, Ashwal S, Barth J, Getchius TS, Gioia GA, et al. Summary of evidence-based guideline update: evaluation and management of concussion in sports Report of the Guideline Development Subcommittee of the American Academy of Neurology. Neurology. 2013;80(24):2250-7.

13. Kara S, Crosswell H, Forch K, Cavadino A, McGeown J, Fulcher M. Less than half of patients recover within 2 weeks of injury after a sports-related mild traumatic brain injury: a 2-year prospective study. Clin J Sport Med. 2020;30(2):96-101.

14. King D, Hume P, Clark T, Pearce A. Use of the King-Devick test for the identification of concussion in an amateur domestic women's rugby union team over two competition seasons in New Zealand. J Neurol Sci. 2020;418:117162.

15. Mathema P, Evans D, Moore IS, Ranson C, Martin R. Concussed or not? An assessment of concussion experience and knowledge within elite and semiprofessional rugby union. Clin J Sport Med. 2016;26(4):320-5.

16. McLeod TCV, Lewis JH, Whelihan K, Bacon CEW. Rest and return to activity after sport-related concussion: a systematic review of the literature. J Athl Train. 2017;52(3):262-87.

17. Dijkstra HP, Pollock N, Chakraverty R, Ardern CL. Return to play in elite sport: a shared decision-making process. Br J Sports Med. 2017;51:419-20.

18. Covassin T, Elbin R, Nakayama Y. Tracking neurocognitive performance following concussion in high school athletes. Phys Sportsmed. 2010;38(4):87-93.

19. Yasen AL, Howell DR, Chou L-S, Pazzaglia AM, Christie AD. Cortical and physical function after mild traumatic brain injury. Med Sci Sports Exerc. 2017;49(6):1066-71.

20. D'Lauro C, Johnson BR, McGinty G, Allred CD, Campbell DE, Jackson JC. Reconsidering return-to-play times: a broader perspective on concussion recovery. Orthop J Sports Med. 2018;6(3):2325967118760854.

21. Twomey C. AFL doubles mandatory break under strict new concussion protocols. Australian Football League. 2021. https:// www.afl.com.au/news/543220/afl- introduces-mandatory-12day-breaks-under-strict-new-concussionprotocols. Accessed 5 Feb 2021.

22. Australian Football League. AFL statement on updated concussion guidelines. 2021. https://www.afl.com.au/news/543223/aflstatement-on-updated-concussion-guidelines. Accessed $20 \mathrm{Apr}$ 2021. 